\title{
Physiological, hematological and
} biochemical factors associated with highaltitude headache in young Chinese males following acute exposure at $3700 \mathrm{~m}$

Kun Wang ${ }^{1,3}$, Menghan Zhang ${ }^{2,3}$, Yi Li $i^{2,3,6}$, Weilin Pu², Yanyun Ma 2,3,6, Yi Wang ${ }^{2,3}$, Xiaoyu Liu ${ }^{2,3}$, Longli Kang ${ }^{5}$, Xiaofeng Wang ${ }^{1,3}$, Jiucun Wang ${ }^{1,3,6}$, Bin Qiao ${ }^{4}$ and Li Jin $^{1,3,6^{*}}$

\begin{abstract}
Background: High-altitude headache $(\mathrm{HAH})$ is the most common sickness occurred in healthy people after rapid ascending to high altitude, and its risk factors were still not well understood. To investigate physiological, hematological and biochemical risk factors associated with high-altitude headache $(\mathrm{HAH})$ after acute exposure to $3700 \mathrm{~m}$, we conducted a two-stage, perspective observational study. In $72 \mathrm{~h}$, total 318 young Han Chinese males ascended from sea level (altitude of $50 \mathrm{~m}$ ) to altitude of $3700 \mathrm{~m}$ by train. Demographic data, physiological, hematological and biochemical parameters of all participants were collected within one week prior to the departure, and within $24 \mathrm{~h}$ after arrival.

Results: The incidence of HAH was $74.84 \%$. For parameters measured at sea level, participants with HAH exhibited significantly higher age and lower BUN ( $p<0.05)$. For parameters measured at $3700 \mathrm{~m}$, participants with $\mathrm{HAH}$ exhibited significantly lower blood oxygen saturation $\left(\mathrm{SpO}_{2}\right)$, higher resting heart rate (HR), higher systolic blood pressure at resting (SBP) and lower blood urea nitrogen (BUN) (all $p<0.05$ ). At $3700 \mathrm{~m}$, the severity of HAH associated with $\mathrm{SpO}_{2}, \mathrm{HR}$ and BUN significantly (all $\mathrm{p}<0.05$ ). Multivariate logistic regression revealed that for parameters at sea level, BUN was associated with HAH [BUN (OR:0.77, 95\% Cl:0.60-0.99)] and for parameters at $3700 \mathrm{~m}, \mathrm{SpO}_{2}, \mathrm{HR}$ and BUN were associated with HAH independently [SpO 2 (OR:0.84, 95\% Cl:0.76-0.93); HR (OR:1.03, 95\% Cl:1.00-1.07); BUN (OR:0.64, 95\% Cl:0.46-0.88)]. No association between hematological parameters and $\mathrm{HAH}$ was observed.

Conclusion: We confirmed that higher $\mathrm{HR}$, lower $\mathrm{SpO}_{2}$ are independent risk factors for $\mathrm{HAH}$. Furthermore, we found that at both $50 \mathrm{~m}$ and $3700 \mathrm{~m}$, lower BUN is a novel independent risk factor for $\mathrm{HAH}$, providing new insights for understanding the pathological mechanisms.
\end{abstract}

Keywords: High-altitude headache, Hypoxia, Blood urea nitrogen, Oxygen saturation, Heart rate

\footnotetext{
* Correspondence: lijin@fudan.edu.cn

'State Key Laboratory of Genetic Engineering, Collaborative Innovation

Center for Genetics and Development, School of Life Sciences, Fudan University, Shanghai 200438, China

${ }^{3}$ Human Phenome Institute, Fudan University, Shanghai 201203, China

Full list of author information is available at the end of the article
}

(c) The Author(s). 2018 Open Access This article is distributed under the terms of the Creative Commons Attribution 4.0 International License (http://creativecommons.org/licenses/by/4.0/), which permits unrestricted use, distribution, and reproduction in any medium, provided you give appropriate credit to the original author(s) and the source, provide a link to the Creative Commons license, and indicate if changes were made. 


\section{Background}

For lowlanders who rapidly ascend to altitude above $2500 \mathrm{~m}$, headache has been considered as the most frequent complaint [1, 2]. According to the most widely-accepted diagnose criteria, the Lake Louise Consensus scoring system identified headache as the cardinal symptom of acute mountain sickness (AMS) [3]. The International Headache Society defined high altitude headache (HAH) as a headache that develops within $24 \mathrm{~h}$ of ascent to high altitude and resolves within $8 \mathrm{~h}$ of descent $[4,5]$. Previous studies have reported that the incidence of $\mathrm{HAH}$ is over $70 \%$ within $24 \mathrm{~h}$ after rapidly ascending above $2500 \mathrm{~m}$ [2].

Numerous studies have been emerged on epidemiology, clinical characteristics, pathophysiological mechanisms, treatment and risk factors of $\mathrm{HAH}[1,6-8]$. Recent studies suggested that the cause of $\mathrm{HAH}$ may be hypoxia-induced cerebral cytotoxic oedema, brain swelling and increased intracranial pressure [6]. In addition to oxygen inhalation, aspirin and acetaminophen were often used for $\mathrm{HAH}$ treatment, and the effect are contradictory $[9,10]$. Several studies have demonstrated that young age, smoking history, higher body mass index history of migraine, high heart rate (HR) and low pulse oxygen saturation $\left(\mathrm{SpO}_{2}\right)$ are independent risk factors for $\mathrm{HAH}[1,11-13]$. Previously, Huang et al. performed the first investigation on the relationship between hematological parameters and $\mathrm{HAH}$ before and after rapid ascending at $3700 \mathrm{~m}$ with 45 subjects, and found that HAH is associated with sea-level reticulocyte and neutrophil counts [14]. Because erythrocytes are the principal carrier of oxygen in the circulatory system, the hematological parameters may provide useful information regarding HAH. Moreover, some studies demonstrated that fluid retention is an important feature of AMS, but other studies have demonstrated that low fluid intake is an independent risk factor of $\mathrm{HAH}$, and glomerular filtration rate estimates increases with AMS severities after rapid ascent to high altitude [15-18]. Over all, most of the studies had small sample sizes, the results were contradictory, and no definitive clear answer is available.

The present study was based on the hypothesis that some hematological and biochemical parameters would be related with HAH. We aimed to explore the association between physiological, hematological, biochemical parameters (including renal function parameters) and the risk of $\mathrm{HAH}$ after a 3-days ascending to the altitude of $3700 \mathrm{~m}$. We carried out a repeated measurement design based on two phases $(50 \mathrm{~m}$ and $3700 \mathrm{~m}$ ) before and after ascending at high altitude for 318 healthy young Han Chinese males. Physical, hematological and biochemical parameters were collected at each phase, respectively.

\section{Methods}

\section{Participants}

In total, 318 young Chinese males who lived at $50 \mathrm{~m}$ and ascended to Tibet for physical training were recruited in this study. All participants reported their disease history, medication history, smoking and drinking history in structured case report forms (CRFs). The inclusion criteria were healthy 18-35 year old Han Chinese men whose primary residence was at an altitude of $\leq 1000 \mathrm{~m}$ and had no high-altitude exposure in recent 2 years. The exclusion criteria were cardio-cerebrovascular diseases, neurological and psychiatric diseases, episodic or chronic migraine diseases or chronic headache symptoms (any headache occurring on more than 2 weeks/mouth), autoimmune diseases, respiratory diseases, malignancy, liver and kidney dysfunction, active infection or a bad cold. Participants who took acetazolamide, diuretics, steroids or nonsteroidal anti-inflammatory drugs during the ascending were excluded. Subjects who agreed to participate underwent a short instruction and explanation of the purpose of this study, and all participants have signed the informed consent before their first examinations. The study was approved by the Human Ethics Committee of Fudan University.

\section{Study procedures and measurements}

All participants ascended to altitude of $3700 \mathrm{~m}$ (Lhasa, Tibet) within $72 \mathrm{~h}$ by train from sea level (altitude of $50 \mathrm{~m}$, Henan). The baseline physiological, hematological and biochemical measurements were performed in the morning, one week prior to the departure at $50 \mathrm{~m}$. Within $24 \mathrm{~h}$ after their arrival at $3700 \mathrm{~m}$, the participants underwent assessments of their physiological, hematological and biochemical parameters, as well as HAH. All participants were monitored by trained physicians for any signs of high-altitude cerebral or pulmonary edema, and immediate treatment will be addressed for emergent cases [19]. During the period of study, all participants maintained the same diet. Caffeine beverage consumption, alcohol consumption and medication use were prohibited, smoking and heavy exercises or physical labor were also avoided.

Structured case report forms (CRFs) were used to record the demographic data (age, body mass index (BMI), chest circumstance, smoking and drinking history) and all measurements at sea level and $3700 \mathrm{~m}$ for each participants. After the acute exposure at $3700 \mathrm{~m}$, the physicians scored HAH based on self-description of patients $(0=$ no headache; $1=$ mild headache; $2=$ moderate headache; 3 = severe headache) was recorded, and the time and place of headache onset was recorded retrospectively. We used a two-repeated measurement method to collect the heart rate at resting ( $\mathrm{HR}$, beats/min), systolic blood pressures at resting (SBP, $\mathrm{mmHg})$, diastolic blood 
pressure at resting (DBP, $\mathrm{mmHg}$ ) and Oxygen saturation $\left(\mathrm{SpO}_{2}, \%\right)$, operated twice by two independent professional physicians and recorded the average value. SBP and DBP were measured using a standardized mercury sphygmomanometer, while $\mathrm{SpO}_{2}$ was measured using Nellcor NPB-40 (USA).

Morning fasting venous blood $(4 \mathrm{ml})$ was collected with EDTA-K2 at both sea level and $3700 \mathrm{~m}$, and $2 \mathrm{ml}$ of the samples were used to assay blood cell parameters by a hematology analyzer (Sysmex pocH-100i, Japan) within $2 \mathrm{~h}$. The hematological parameters included red blood cell count (RBC, $\left.\times 10^{12} / \mathrm{L}\right)$, hemoglobin (Hgb, g/ L), hematocrit (Hct, \%), mean corpuscular volume (MCV, fL), mean corpuscular hemoglobin $(\mathrm{MCH}, \mathrm{pg})$, white blood cell count (WBC, $\times 10^{9} / \mathrm{L}$ ), lymphocyte percentage (LYM\%), absolute lymphocyte count (LYM\#), platelet count (PLT, $\times 10^{9} / \mathrm{L}$ ) and mean platelet volume (MPV, fL). The rest $2 \mathrm{ml}$ blood specimens were centrifuged $3000 \mathrm{r} / \mathrm{min}$ for 10 minus to separate plasma. Biochemical parameters including alanine aminotransferase (ALT, U/L), aspartate aminotransferase (AST, U/L), blood urea nitrogen (BUN, $\mathrm{mmol} / \mathrm{L}$ ), serum creatinine (CREA, umol/L), total serum bilirubin (T-BIL, umol/L), direct serum bilirubin (D-BIL, umol/
L) and indirect serum bilirubin (I-BIL, umol/L) were measured using automatic biochemistry analyzer (TOSHIBA TBA-120FR).

The diagnosis of $\mathrm{HAH}$ was based on the International Classification of Headache Disorders $3 \beta$ criteria [5], but not strictly (no test for descending), restricted by the complex situations of field study.

\section{Statistical analyses}

The normality of continuous data was assessed by Shapiro-Wilk's test. Normally distributed data were presented as the means \pm standard deviations (SD), non-normally distributed data were presented as median (interquartile range, IQR) and enumerated data were expressed as numbers (\%). The differences of measurements between sea level and $3700 \mathrm{~m}$ were compared using paired-sample student's t-test for normally distributed data, and the comparisons of differences between $\mathrm{HAH}$ positive $(\mathrm{HAH}+)$ and $\mathrm{HAH}$ negative (HAH-) groups were analyzed by independent student's t-test. The non-normally data were compared using the Mann-Whitney U test. The spearman correlations between $\mathrm{HAH}$ score and the measurements at sea level and $3700 \mathrm{~m}$ were analyzed. Univariate logistic

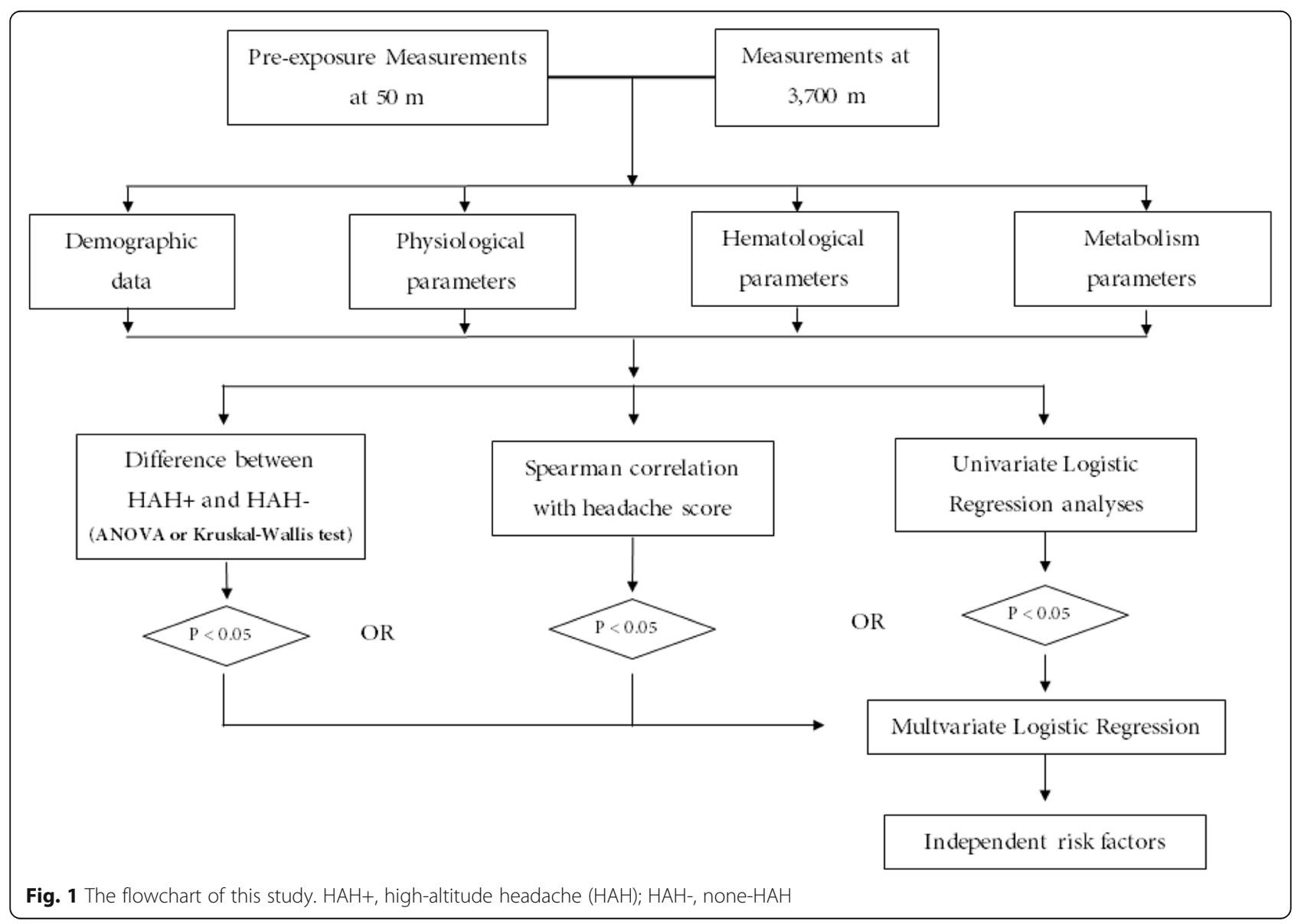


regression was performed to assess the relationships between each measurements and HAH at $3700 \mathrm{~m}$. Significant variables in any of the above three analyses were included in forward stepwise multivariable logistic regression, and analyzed separately at sea level and $3700 \mathrm{~m}$. The significant level of $p$-value is 0.05 (two-tailed). The analyses flowchart was shown in Fig. 1.

\section{Results}

Total 318 participants had complete CRFs, physiological, hematological and biochemical measurements
(Additional file 1). The mean age and BMI of the participants in this study were $21.87 \pm 3.33$ years and $22.06 \pm$ $1.99 \mathrm{~kg} / \mathrm{m}^{2}$. The incidence of HAH after acute exposure to $3700 \mathrm{~m}$ is $74.84 \%$ (Additional files 2 and 3 ).

\section{Alterations in physiological, hematological and biochemical measurements}

Most of the physiological, hematological and biochemical parameters were dramatically altered after acute exposure to high altitude from sea level, beside of $\mathrm{Hct}, \mathrm{MCH}$ and LYM\%. For the physiological measurements, $\mathrm{SpO}_{2}$

Table 1 Comparison of physiological, hematological and biochemical parameters between sea level and $3700 \mathrm{~m}(\mathrm{~N}=318)$

\begin{tabular}{|c|c|c|c|}
\hline & Measurements at sea-level & Measurements at $3700 \mathrm{~m}$ & $p$ \\
\hline \multicolumn{4}{|l|}{ Demographic data } \\
\hline Age, y & $21.87 \pm 3.33$ & the same as sea-level & - \\
\hline $\mathrm{BMI}, \mathrm{kg} / \mathrm{m}^{2}$ & $22.06 \pm 1.99$ & the same as sea-level & - \\
\hline chest circumstance, $\mathrm{cm}$ & $86.11 \pm 5.09$ & the same as sea-level & - \\
\hline Smoking, yes(\%) & $102(32.1)$ & the same as sea-level & - \\
\hline Drinking, yes(\%) & $39(12.3)$ & the same as sea-level & - \\
\hline \multicolumn{4}{|l|}{ Physical parameters } \\
\hline $\mathrm{SpO}_{2}, \%$ & $98.00(98.00-98.20)$ & $88.00(85.10-90.20)$ & $<0.001^{* *}$ \\
\hline $\mathrm{HR}$, beats/min & $67.49 \pm 9.18$ & $84.17 \pm 12.15$ & $<0.001^{* *}$ \\
\hline $\mathrm{SBP}, \mathrm{mmHg}$ & $111.22 \pm 9.81$ & $120.52 \pm 12.8$ & $<0.001^{* *}$ \\
\hline $\mathrm{DBP}, \mathrm{mmHg}$ & $71.85 \pm 8.14$ & $81.77 \pm 9.56$ & $<0.001^{* *}$ \\
\hline \multicolumn{4}{|l|}{ Hematological parameters } \\
\hline $\mathrm{RBC}, * 10^{12}$ & $5.00(4.80-5.20)$ & $5.11(4.82-5.49)$ & $<0.001^{* *}$ \\
\hline $\mathrm{Hgb}, \mathrm{g} / \mathrm{L}$ & $151.10(144.00-157.00)$ & $157.00(147.00-169.30)$ & $<0.001^{* *}$ \\
\hline $\mathrm{Hct}, \%$ & $44.49 \pm 3.10$ & $48.42 \pm 6.08$ & $<0.001^{* *}$ \\
\hline$M C V, f L$ & $92.20(90.00-94.22)$ & $86.50(84.40-88.62)$ & $<0.001^{* *}$ \\
\hline $\mathrm{MCH}, \mathrm{pg}$ & $30.59(29.61-31.30)$ & $30.90(30.20-32.10)$ & $<0.001^{* *}$ \\
\hline $\mathrm{PLT},{ }^{*} 10^{9}$ & $206.79 \pm 44.77$ & $244.97 \pm 69.80$ & $<0.001^{* *}$ \\
\hline$M P V, f L$ & $10.50(9.80-11.00)$ & $10.60(10.20-11.00)$ & $0.006^{* *}$ \\
\hline WBC, $* 10^{9}$ & $6.10(5.40-7.10)$ & $7.90(7.00-9.50)$ & $<0.001^{* *}$ \\
\hline LYM\%, \% & $36.23 \pm 7.59$ & $36.49 \pm 9.06$ & 0.833 \\
\hline LYM\#, ${ }^{*} 10^{9}$ & $2.20(1.90-2.60)$ & $2.95(2.50-3.40)$ & $<0.001^{* *}$ \\
\hline \multicolumn{4}{|l|}{ Biochemical parameters } \\
\hline $\mathrm{ALT}, \mathrm{U} / \mathrm{L}$ & $18.00(15.00-23.00)$ & $39.20(30.40-48.50)$ & $<0.001^{* *}$ \\
\hline AST, U/L & $15.10(12.30-18.50)$ & $31.00(25.50-40.60)$ & $<0.001^{* *}$ \\
\hline $\mathrm{BUN}, \mathrm{mmol} / \mathrm{L}$ & $4.45(3.89-5.28)$ & $4.60(4.10-5.25)$ & 0.109 \\
\hline CREA, umol/L & $58.00(49.00-68.10)$ & $105.40(98.60-114.00)$ & $<0.001^{* *}$ \\
\hline TBIL, umol/L & $12.60(10.80-14.00)$ & $12.30(10.20-15.65)$ & $0.023^{*}$ \\
\hline DBIL, umol/L & $3.10(2.30-3.50)$ & $4.70(3.90-5.80)$ & $<0.001^{* *}$ \\
\hline $\mid \mathrm{BIL}, \mathrm{umol} / \mathrm{L}$ & $9.70(8.50-10.50)$ & $7.70(5.80-10.65)$ & $<0.001^{* *}$ \\
\hline
\end{tabular}

$\mathrm{SpO}_{2}$ blood oxygen saturation, $H R$ heart rate at resting, SBP systolic blood pressure, DBP diastolic blood pressure, RBC red blood cell count, $\mathrm{Hgb}$ hemoglobin, $\mathrm{Hct}$ hematocrit, MCV mean corpuscular volume, MCH mean corpuscular hemoglobin, PLT platelets count, MPV mean platelet volume, WBC white blood cell, $L Y M \%$ lymphocyte rate, $L Y M \#$ lymphocyte count, $A L T$ alanine aminotransferase, $A S T$ aspartate aminotransferase, BUN blood urea nitrogen, CREA creatinine, TBIL total serum bilirubin, $D B I L$ direct serum bilirubin, $I B I L$ indirect serum bilirubin Normally distributed variables were presented as mean $\pm S D$, and compared using paired-sample $T$ test; Non-normally distributed variables were presented as mean (interquartile range), and compared using Mann-Whitney $\mathrm{U}$ test ${ }^{*} p$ value indicates $p<0.05 ;{ }^{* *} p$ value indicates $p<0.01$ 
decreased from 98.00 (IQR, 98.00-98.20) to 88.00 (IQR, 85.10-90.20) $(p<0.01)$, while HR, SBP and DBP significantly increased from sea level to $3700 \mathrm{~m}$. For the hematological measurements, RBC, Hgb, Hct, PLT, MCH, MPV, WBC and LYM\# all significantly increased, while MCV showed significant decrease (all $p<0.001$ ). However, the alteration of LYM\% between sea level and $3700 \mathrm{~m}$ was not significant. For the biochemical measurements, ALT,
AST, CREA, TBIL, DBIL and IBIL exhibited a significant increase, while BUN had no significant difference between sea level and $3700 \mathrm{~m}$. (Table 1).

\section{Comparison of physiological, hematological and biochemical parameters between the $\mathrm{HAH}+$ and $\mathrm{HAH}$ - groups}

Regarding the measurements at sea level, compared to the $\mathrm{HAH}$ - group, the $\mathrm{HAH}+$ group had significant higher age

Table 2 Difference between $\mathrm{HAH}+$ and $\mathrm{HAH}$ - subjects in demographics, physiological, hematological and biochemical parameters at sea level and $3700 \mathrm{~m}$

\begin{tabular}{|c|c|c|c|c|c|c|}
\hline & \multicolumn{3}{|c|}{ Measurements at sea level } & \multicolumn{3}{|l|}{ Measurements at $3700 \mathrm{~m}$} \\
\hline & $\mathrm{HAH}+(n=238)$ & $\mathrm{HAH}-(n=80)$ & p & $\mathrm{HAH}+(n=238)$ & $\mathrm{HAH}-(n=80)$ & $p$ \\
\hline \multicolumn{7}{|l|}{ Demographic data } \\
\hline Age (year) & $22.05 \pm 3.53$ & $21.30 \pm 2.69$ & $0.046^{*}$ & The same as sea-levels & & \\
\hline $\mathrm{BMI}\left(\mathrm{kg} / \mathrm{m}^{2}\right)$ & $22.08 \pm 2.02$ & $21.96 \pm 1.86$ & 0.662 & The same as sea-levels & & \\
\hline Chest Circumstance $(\mathrm{cm})$ & $86.19 \pm 5.30$ & $86.04 \pm 4.59$ & 0.813 & The same as sea-levels & & \\
\hline Smoking (yes, \%) & $73(30.7)$ & $29(36.3)$ & 0.417 & The same as sea-levels & & \\
\hline Drinking (yes, \%) & $29(12.2)$ & $10(12.5)$ & 0.978 & The same as sea-levels & & \\
\hline \multicolumn{7}{|l|}{ Physiological parameters } \\
\hline $\mathrm{SpO}_{2}(\%)$ & 98.00 (97.90-98.00) & 98.00 (98.00-98.00) & 0.171 & 88.00 (84.90-90.40) & 90.00 (87.30-92.10) & $<0.001^{* *}$ \\
\hline HR (beats/min) & $67.72 \pm 8.99$ & $66.91 \pm 9.86$ & 0.522 & $85.30 \pm 12.09$ & $80.77 \pm 11.75$ & $<0.001^{* *}$ \\
\hline $\mathrm{SBP}(\mathrm{mmHg})$ & $110.97 \pm 10.09$ & $111.98 \pm 8.88$ & 0.405 & $119.74 \pm 13.10$ & $123.41 \pm 11.10$ & $0.046^{*}$ \\
\hline $\mathrm{DBP}(\mathrm{mmHg})$ & $71.69 \pm 8.23$ & $72.30 \pm 7.91$ & 0.569 & $81.42 \pm 9.86$ & $82.99 \pm 8.39$ & 0.251 \\
\hline \multicolumn{7}{|l|}{ Hematological parameters } \\
\hline $\operatorname{RBC}\left({ }^{*} 10^{12}\right)$ & $5.00(4.80-5.20)$ & $5.00(4.70-5.20)$ & 0.559 & $5.10(4.83-5.48)$ & $5.11(4.75-5.55)$ & 0.955 \\
\hline $\mathrm{Hgb}(\mathrm{g} / \mathrm{L})$ & $151.30(144.00-157.00)$ & $150.00(143.80-158.10)$ & 0.945 & $157.00(149.60-168.30)$ & $157.00(144.50-170.40)$ & 0.641 \\
\hline Hct (\%) & $45.69 \pm 2.46$ & $45.70 \pm 2.49$ & 0.981 & $48.61 \pm 5.95$ & $47.83 \pm 6.47$ & 0.364 \\
\hline MCV (fL) & $92.30(90.00-94.75)$ & $92.00(90.20-94.10)$ & 0.598 & $86.50(84.40-88.92)$ & $86.40(84.30-87.75)$ & 0.336 \\
\hline $\mathrm{MCH}(\mathrm{pg})$ & $30.60(29.59-31.36)$ & $30.59(29.73-31.02)$ & 0.459 & $30.90(29.95-32.10)$ & $31.00(30.18-31.75)$ & 0.939 \\
\hline $\operatorname{PLT}\left({ }^{*} 10^{9}\right)$ & $207.95 \pm 44.97$ & $203.33 \pm 44.27$ & 0.438 & $244.8 \pm 68.6$ & $245.3 \pm 73.8$ & 0.960 \\
\hline MPV (fL) & $10.50(9.80-11.02)$ & $10.40(9.70-10.91)$ & 0.326 & $10.60(10.20-11.03)$ & $10.60(10.30-11.00)$ & 0.965 \\
\hline WBC $\left({ }^{*} 10^{9}\right)$ & $6.00(5.40-7.00)$ & $6.50(5.50-7.35)$ & 0.090 & $7.90(7.10-9.50)$ & 7.95 (6.98-9.38) & 0.791 \\
\hline LYM rate (\%) & $36.60 \pm 7.58$ & $35.12 \pm 7.56$ & 0.144 & $36.53 \pm 9.68$ & $36.36 \pm 6.89$ & 0.871 \\
\hline LYM count $\left({ }^{*} 10^{9}\right)$ & $2.20(1.90-2.60)$ & $2.15(1.90-2.60)$ & 0.703 & $3.00(2.51-3.40)$ & $2.95(2.58-3.50)$ & 0.910 \\
\hline \multicolumn{7}{|l|}{ Biochemical parameters } \\
\hline $\operatorname{ALT}(\mathrm{U} / \mathrm{L})$ & $18.10(15.00-24.10)$ & $18.00(14.00-21.00)$ & 0.187 & $39.20(28.50-49.30)$ & $39.00(29.30-46.80)$ & 0.563 \\
\hline $\mathrm{AST}(\mathrm{U} / \mathrm{L})$ & $15.20(12.00-20.00)$ & 14.00 (12.10-17.75) & 0.237 & $31.00(25.00-40.25)$ & $32.00(27.20-38.50)$ & 0.581 \\
\hline BUN (mmol/L) & $4.38(3.82-5.08)$ & 4.69 (3.99-5.53) & $0.029^{*}$ & $4.50(4.02-5.20)$ & $4.80(4.40-5.40)$ & $0.003^{* *}$ \\
\hline CREA (umol/L) & $58.00(49.00-65.00)$ & $59.00(50.10-68.20)$ & 0.082 & $105.30(98.50-115.00)$ & 106.80 (99.70-113.30) & 0.811 \\
\hline TBIL (umol/L) & $12.50(10.80-14.00)$ & $12.70(10.85-14.00)$ & 0.876 & $12.75(10.20-15.75)$ & $12.90(10.20-15.50)$ & 0.709 \\
\hline DBIL (umol/L) & $3.10(2.30-3.50)$ & $3.10(2.15-3.50)$ & 0.553 & 4.85 (3.90-5.90) & $4.60(3.80-5.80)$ & 0.274 \\
\hline IBIL (umol/L) & $9.60(8.50-10.43)$ & $9.90(8.50-10.55)$ & 0.933 & $7.60(5.80-10.70)$ & $7.80(6.00-10.40)$ & 0.877 \\
\hline
\end{tabular}

$\mathrm{HAH}$ high altitude headache, $\mathrm{HAH}+$ with high-altitude headache, $\mathrm{HAH}$ - without high-altitude headache, $\mathrm{SpO}_{2}$ blood oxygen saturation, $\mathrm{HR}$ heart rate at resting, $\mathrm{SBP}$ systolic blood pressure, DBP diastolic blood pressure, $R B C$ red blood cell count, Hgb hemoglobin, Hct hematocrit, $M C V$ mean corpuscular volume, $M C H$ mean corpuscular hemoglobin, PLT platelets count, MPV mean platelet volume, WBC white blood cell, $L Y M \%$ lymphocyte rate, $L Y M \#$ lymphocyte count, $A L T$ alanine aminotransferase, $A S T$ aspartate aminotransferase, $B U N$ blood urea nitrogen, $C R E A$ creatinine, $T B I L$ total serum bilirubin, $D B I L$ direct serum bilirubin, IBIL indirect serum bilirubin

Normally distributed variables were presented as mean $\pm \mathrm{SD}$, and compared using student's t-test; Non-normally distributed variables were presented as mean (interquartile range), and compared using Mann-Whitney $\mathrm{U}$ test; Categorical variables were compared using fisher's exact test;

${ }^{*} p$ value indicates $p<0.05 ;{ }^{* *} p$ value indicates $p<0.01$ 
$(\mathrm{HAH}+: 22.05 \pm 3.53$ vs $\mathrm{HAH}-: 21.30 \pm 2.69, p=0.046)$ and significant lower BUN (HAH+: $4.38(3.82-5.08)$ vs HAH-: 4.69 (3.99-5.53), $p=0.029)$. There were no significant differences in the physiological and hematological measurements at sea level (all $p>0.05$, Table 2).

Regarding the measurements at $3700 \mathrm{~m}$, compared with the $\mathrm{HAH}$ - group, the $\mathrm{HAH}+$ group had significant lower $\mathrm{SpO}_{2}(\mathrm{HAH}+88.00$ (84.90-90.40) vs HAH- 90.00 (87.30-92.10), $p<0.001)$, lower SBP $(\mathrm{HAH}+119.74 \pm$ 13.10 vs $\mathrm{HAH}-123.41 \pm 11.10, \mathrm{p}=0.046)$ and lower BUN (HAH+ 4.50 (4.02-5.20) vs HAH- 4.80 (4.40-5.40), $p=0.003)$. The $\mathrm{HAH}+$ group also had significant higher $\mathrm{HR}$ than $\mathrm{HAH}$ - group $(\mathrm{HAH}+85.30 \pm 12.09$ vs $\mathrm{HAH}$ $80.77 \pm 11.75, \mathrm{p}<0.001)$. The hematological measurements showed no significant differences between HAH+ and $\mathrm{HAH}$ - groups at $3700 \mathrm{~m}$ (all $p>0.05$, Table 2).

\section{Relationship between physiological, hematological and biochemical parameters and $\mathrm{HAH}$}

We further used Spearman's correlation analyses to explore the relationship between measurements and the HAH severity. For the measurements at sea level, no significant association was observed between demographic data or all measurements and $\mathrm{HAH}$ severity. For the measurements at $3700 \mathrm{~m}$, HAH severity was significantly associated with $\mathrm{SpO}_{2}(r=-0.365, p<0.001)$, HR $(r=0.249, p=0.002)$ and BUN $(r=-0.176, p=0.006)$. The hematological measurements showed no significant correlation with HAH severity (all $\mathrm{p}>0.05$, Table 3 ).

\section{Risk factors for HAH at sea level and $3700 \mathrm{~m}$}

To discover risk factors for $\mathrm{HAH}$, we performed univariate logistic regression for all parameters at sea level and at $3700 \mathrm{~m}$. Among the sea level parameters, the univariate logistic regression revealed that only BUN associated with HAH significantly (OR:0.78, 95\% CI:0.61$0.99, p=0.044)$. Meanwhile, among the parameters at $3700 \mathrm{~m}, \mathrm{SpO}_{2}$ (OR:0.84, 95\% CI:0.77-0.90, $\left.p<0.001\right)$, HR (OR:1.03, 95\% CI:1.01-1.06, $p=0.004$ ) and BUN (OR:0.72, 95\% CI:0.55-0.93, $p=0.012$ ) exhibited significant association with HAH (Table 4).

Multivariate logistic regression was performed for parameters which showed significant associations with $\mathrm{HAH}$ in univariate logistic regression, correlated with headache score, or showed significant difference between $\mathrm{HAH}+$ and $\mathrm{HAH}$ - groups. For parameters at sea level, only BUN showed significant protective effect on HAH (OR:0.77, 95\% CI:0.60-0.99, $p=0.040$ ), but for parameters at $3700 \mathrm{~m}$, multivariate logistic regression revealed that $\mathrm{SpO}_{2}$ (OR:0.84, 95\% CI:0.76-0.93, $p<0.001$ ), HR (OR:1.03, 95\% CI:1.00-1.07, $p=0.042$ ) and BUN (OR:0.64, 95\% CI:0.46-0.88, $p=0.007$ ) were all independent risk factors for HAH (Table 5).
Table 3 Relationships between HAH score and all the parameters at both sea level and $3700 \mathrm{~m}(\mathrm{~N}=318)$

\begin{tabular}{|c|c|c|c|c|}
\hline & \multicolumn{2}{|c|}{$\begin{array}{l}\text { Measurements } \\
\text { at sea-level }\end{array}$} & \multicolumn{2}{|c|}{$\begin{array}{l}\text { Measurements } \\
\text { at } 3700 \mathrm{~m}\end{array}$} \\
\hline & $\begin{array}{l}\text { With HAH } \\
\text { score r }\end{array}$ & $p$ & $\begin{array}{l}\text { With HAH } \\
\text { score r }\end{array}$ & $p$ \\
\hline \multicolumn{5}{|l|}{ Demographic data } \\
\hline Age (y) & 0.113 & 0.053 & \multicolumn{2}{|c|}{ The same as sea-level } \\
\hline $\mathrm{BMI}\left(\mathrm{kg} / \mathrm{m}^{2}\right)$ & 0.087 & 0.129 & \multicolumn{2}{|c|}{ The same as sea-level } \\
\hline Chest Circumstance $(\mathrm{cm})$ & 0.079 & 0.187 & \multicolumn{2}{|c|}{ The same as sea-level } \\
\hline \multicolumn{5}{|l|}{ Physical parameters } \\
\hline $\mathrm{SpO}_{2}(\%)$ & -0.085 & 0.168 & -0.365 & $<0.001^{* *}$ \\
\hline HR (beats/min) & 0.003 & 0.859 & 0.249 & $0.002^{* *}$ \\
\hline $\mathrm{SBP}(\mathrm{mmHg})$ & -0.049 & 0.471 & -0.050 & 0.518 \\
\hline $\mathrm{DBP}(\mathrm{mmHg})$ & -0.073 & 0.246 & -0.008 & 0.865 \\
\hline \multicolumn{5}{|l|}{ Metabolic parameters } \\
\hline $\mathrm{RBC}\left({ }^{*} 10^{12}\right)$ & 0.013 & 0.984 & 0.041 & 0.468 \\
\hline $\mathrm{HGB}(\mathrm{g} / \mathrm{L})$ & 0.008 & 0.910 & 0.046 & 0.358 \\
\hline $\mathrm{HCT}(\%)$ & 0.015 & 0.976 & 0.056 & 0.333 \\
\hline MCV (fL) & -0.012 & 0.927 & -0.008 & 0.620 \\
\hline $\mathrm{MCH}(\mathrm{pg})$ & 0.020 & 0.640 & -0.042 & 0.941 \\
\hline $\operatorname{PLT}\left({ }^{*} 10^{9}\right)$ & 0.057 & 0.336 & -0.021 & 0.420 \\
\hline MPV (fL) & 0.077 & 0.428 & -0.023 & 0.871 \\
\hline WBC $\left({ }^{*} 10^{9}\right)$ & -0.102 & 0.131 & 0.081 & 0.900 \\
\hline LYM\% (\%) & 0.024 & 0.688 & -0.061 & 0.341 \\
\hline LYM\# $\left({ }^{*} 10^{9}\right)$ & 0.035 & 0.687 & 0.017 & 0.278 \\
\hline \multicolumn{5}{|l|}{ Biochemical parameters } \\
\hline $\mathrm{ALT}(\mathrm{U} / \mathrm{L})$ & 0.072 & 0.388 & 0.056 & 0.987 \\
\hline AST (U/L) & 0.065 & 0.446 & -0.051 & 0.100 \\
\hline BUN (mmol/L) & -0.071 & 0.211 & -0.176 & $0.006^{* *}$ \\
\hline CREA (umol/L) & -0.045 & 0.425 & 0.009 & 0.759 \\
\hline TBIL (umol/L) & -0.004 & 0.845 & 0.105 & 0.657 \\
\hline DBIL (umol/L) & 0.037 & 0.581 & 0.034 & 0.672 \\
\hline IBIL (umol/L) & -0.027 & 0.539 & 0.106 & 0.583 \\
\hline
\end{tabular}

$\mathrm{HAH}$ high altitude headache, $\mathrm{SpO}_{2}$ blood oxygen saturation, $\mathrm{HR}$ heart rate at resting, $S B P$ systolic blood pressure, $D B P$ diastolic blood pressure, $R B C$ red blood cell count, $\mathrm{Hgb}$ hemoglobin, $\mathrm{Hct}$ hematocrit, $\mathrm{MCV}$ mean corpuscular volume, $\mathrm{MCH}$ mean corpuscular hemoglobin, $P L T$ platelets count, MPV mean platelet volume, WBC white blood cell, LYM\% lymphocyte rate, LYM\# lymphocyte count, $A L T$ alanine aminotransferase, AST aspartate aminotransferase, BUN blood urea nitrogen, CREA creatinine, TBIL total serum bilirubin, $D B I L$ direct serum bilirubin, $I B I L$ indirect serum bilirubin ${ }^{*} p$ value indicates $p<0.05$; ${ }^{* *} p$ value indicates $p<0.01$

\section{Discussion}

Alterations in physiological, hematological and biochemical parameters

Our study identified alterations in physiological, hematological and biochemical parameters from sea level to altitude of $3700 \mathrm{~m}$. High altitude hypoxia lead a reduction of $\mathrm{SpO}_{2}$, which may result in a decrease in 
Table 4 Univariate logistic regression for each measurements at sea level and $3700 \mathrm{~m}(N=318)$

\begin{tabular}{|c|c|c|c|c|c|c|c|c|}
\hline & \multicolumn{4}{|c|}{ Measurements at sea level } & \multicolumn{4}{|c|}{ Measurements at $3700 \mathrm{~m}$} \\
\hline & $\beta$-coefficient & OR & $95 \% \mathrm{Cl}$ & $p$ & $\beta$-coefficient & OR & $95 \% \mathrm{Cl}$ & $p$ \\
\hline \multicolumn{9}{|l|}{ Demographic Data } \\
\hline age (y) & 0.076 & 1.08 & $0.99-1.19$ & 0.090 & \multicolumn{4}{|c|}{ The same as sea level } \\
\hline BMI (kg/m2) & 0.028 & 1.03 & $0.90-1.18$ & 0.674 & \multicolumn{4}{|c|}{ The same as sea level } \\
\hline Chest (cm) & 0.006 & 1.01 & $0.96-1.06$ & 0.825 & \multicolumn{4}{|c|}{ The same as sea level } \\
\hline smoking & -0.249 & 0.76 & $0.42-1.33$ & 0.453 & \multicolumn{4}{|c|}{ The same as sea level } \\
\hline drinking & -0.055 & 0.97 & $0.45-2.16$ & 0.917 & \multicolumn{4}{|c|}{ The same as sea level } \\
\hline \multicolumn{9}{|c|}{ Physical parameters } \\
\hline $\mathrm{SpO}_{2}(\%)$ & -0.058 & 0.94 & $0.75-1.12$ & 0.555 & -0.176 & 0.84 & $0.77-0.90$ & $<0.001^{* *}$ \\
\hline HR (beats/min) & 0.010 & 1.01 & $0.98-1.04$ & 0.501 & 0.032 & 1.03 & $1.01-1.06$ & $0.004^{* *}$ \\
\hline $\mathrm{SBP}(\mathrm{mmHg})$ & -0.010 & 0.99 & $0.96-1.02$ & 0.433 & -0.022 & 0.98 & $0.95-1.00$ & 0.068 \\
\hline $\mathrm{DBP}(\mathrm{mmHg})$ & -0.009 & 0.99 & $0.96-1.02$ & 0.576 & -0.017 & 0.98 & $0.95-1.01$ & 0.291 \\
\hline \multicolumn{9}{|c|}{ Hematological parameters } \\
\hline $\operatorname{RBC}\left({ }^{*} 10^{12}\right)$ & -0.197 & 0.82 & $0.39-1.76$ & 0.605 & 0.072 & 1.07 & $0.75-1.55$ & 0.696 \\
\hline $\mathrm{HGB}(\mathrm{g} / \mathrm{L})$ & -0.002 & 1.00 & 0.97-1.03 & 0.906 & 0.004 & 1.00 & $0.99-1.02$ & 0.460 \\
\hline HCT (\%) & -0.001 & 1.00 & $0.90-1.11$ & 0.981 & 0.022 & 1.02 & $0.98-1.07$ & 0.341 \\
\hline MCV (fL) & 0.014 & 1.01 & $0.96-1.06$ & 0.580 & 0.020 & 1.02 & $0.98-1.07$ & 0.356 \\
\hline $\mathrm{MCH}(\mathrm{pg})$ & 0.023 & 1.02 & 0.89-1.16 & 0.718 & 0.015 & 1.02 & $0.92-1.13$ & 0.770 \\
\hline $\operatorname{PLT}\left({ }^{*} 10^{9}\right)$ & 0.002 & 1.00 & 0.99-1.01 & 0.439 & 0.000 & 1.00 & 0.99-1.01 & 0.959 \\
\hline MPV (fL) & 0.136 & 1.15 & $0.88-1.51$ & 0.320 & 0.039 & 1.04 & $0.70-1.56$ & 0.847 \\
\hline WBC $\left({ }^{*} 10^{9}\right)$ & -0.153 & 0.86 & $0.71-1.03$ & 0.105 & 0.041 & 1.04 & $0.92-1.19$ & 0.525 \\
\hline LYM\% (\%) & 0.013 & 1.01 & 0.99-1.06 & 0.959 & 0.002 & 1.00 & $0.97-1.03$ & 0.890 \\
\hline LYM\# $\left({ }^{*} 10^{9}\right)$ & 0.026 & 1.03 & $0.61-1.70$ & 0.144 & 0.074 & 1.08 & $0.78-1.50$ & 0.655 \\
\hline \multicolumn{9}{|c|}{ Biochemical parameters } \\
\hline $\mathrm{ALT}(\mathrm{U} / \mathrm{L})$ & 0.027 & 1.03 & $0.99-1.07$ & 0.119 & 0.014 & 1.01 & $0.97-1.07$ & 0.561 \\
\hline AST (U/L) & 0.030 & 1.03 & 0.99-1.08 & 0.180 & 0.005 & 1.00 & $0.98-1.03$ & 0.670 \\
\hline BUN (mmol/L) & -0.252 & 0.78 & $0.61-0.99$ & $0.044^{*}$ & -0.330 & 0.72 & $0.55-0.93$ & $0.012^{*}$ \\
\hline CREA (umol/L) & -0.021 & 0.98 & $0.95-1.00$ & 0.096 & 0.003 & 1.00 & $0.98-1.03$ & 0.823 \\
\hline TBIL (umol/L) & 0.043 & 1.04 & $0.91-1.19$ & 0.531 & 0.026 & 1.03 & $0.97-1.09$ & 0.345 \\
\hline DBIL (umol/L) & 0.161 & 1.17 & $0.79-1.75$ & 0.427 & 0.137 & 1.15 & $0.97-1.37$ & 0.117 \\
\hline IBIL (umol/L) & 0.051 & 1.05 & $0.87-1.28$ & 0.605 & 0.017 & 1.02 & $0.96-1.09$ & 0.599 \\
\hline
\end{tabular}

$\mathrm{SpO}_{2}$ blood oxygen saturation, $\mathrm{HR}$ heart rate at resting, SBP systolic blood pressure, DBP diastolic blood pressure, $R B C$ red blood cell count, $\mathrm{Hgb}$ hemoglobin, $\mathrm{Hct}$ hematocrit, $M C V$ mean corpuscular volume, $M C H$ mean corpuscular hemoglobin, $P L T$ platelets count, MPV mean platelet volume, WBC white blood cell, $L Y M \%$ lymphocyte rate, $L Y M \#$ lymphocyte count, $A L T$ alanine aminotransferase, $A S T$ aspartate aminotransferase, $B U N$ blood urea nitrogen, $C R E A$ creatinine, $T B I L$ total serum bilirubin, $D B I L$ direct serum bilirubin, IBIL indirect serum bilirubin

${ }^{*} p$ value indicates $p<0.05 ;{ }^{* *} p$ value indicates $p<0.01$

the delivery of oxygen and energy to organs and tissues. Dropped blood oxygen level may stimulate carotid chemoreceptors and activate the autonomic nervous system, which results in the cardiac output improvement, finally leading to increased HR [20]. The observation of our study is in consistency with previous studies $[1,7,13]$.

The low humidity, hypoxic tachypnea and reduced fluid intake can lead to insensible fluid lose at high altitude $[15,16]$. In addition, within hours of exposure to high altitude hypoxia, hypoxic tachypnea may lead to respiratory alkalosis, increased natriuresis and diuresis, promoting fluid shift away from intravascular space, result in blood concentration, even hypovolemia [21]. Our study observed most hematological parameters (RBC, Hgb, Hct, MCH, PLT, WBC and LYM\#) were elevated from sea-level to altitude of $3700 \mathrm{~m}$ within a $72 \mathrm{~h}$ ascending processes, which also in consistency with previous studies [14].

Our study also observed that serum creatinine increased dramatically after ascent from sea level to $3700 \mathrm{~m}$, indicating a significantly decreased estimated glomerular filtration 
Table 5 Forward stepwise multivariate logistic regression for $\mathrm{HAH}$ at sea level and $3700 \mathrm{~m}(\mathrm{~N}=318)$

\begin{tabular}{lllll}
\hline & $\beta$-coefficient & OR & $95 \% \mathrm{Cl}$ & $p$-value \\
\hline \multicolumn{4}{l}{ Measurements at sea level (after variable selection) } \\
age & 0.075 & 1.08 & $0.99-1.18$ & 0.092 \\
BUN & -0.259 & 0.77 & $0.60-0.99$ & $0.040^{*}$ \\
Measurements at $3700 \mathrm{~m}$ (after variable selection) & \\
age & 0.090 & 1.09 & $0.98-1.23$ & 0.110 \\
$\mathrm{SpO}_{2}$ & -0.191 & 0.84 & $0.76-0.93$ & $0.001^{* *}$ \\
$\mathrm{HR}$ & 0.029 & 1.03 & $1.00-1.07$ & $0.042^{*}$ \\
$\mathrm{SBP}$ & -0.019 & 0.98 & $0.95-1.01$ & 0.091 \\
$\mathrm{BUN}$ & -0.452 & 0.64 & $0.46-0.88$ & $0.007^{* *}$ \\
\hline
\end{tabular}

$\mathrm{SpO}_{2}$ blood oxygen saturation, $\mathrm{HR}$ heart rate at resting, $\mathrm{SBP}$ systolic blood pressure, $B U N$ blood urea nitrogen

${ }^{*} p$ value indicates $p<0.05 ;{ }^{* *} p$ value indicates $p<0.01$

rate (eGFR). Previous study has suggested that a linear decrease of eGFR with the increase of altitude, which may related to a reduction of renal plasma flow (secondary to the increased sympathetic activity) [18]. In addition, our study found that there was only little change of BUN from sea level to $3700 \mathrm{~m}$, which is inconsistent with previous study [22]. Because both BUN and creatinine were commonly used as renal function markers, we draw a scatter plot to explore the change of the relationship between BUN and creatinine at $50 \mathrm{~m}$ and $3700 \mathrm{~m}$ (See fig. 2). This figure showed that there is a strong linear correlation between BUN and creatinine is at $50 \mathrm{~m}$ altitude, but no significant correlation after ascent to $3700 \mathrm{~m}$. The BUN level represent the urea concentration of plasma, which can be reabsorbed in inner medullary collecting duct (IMCD), while creatinine can not be reabsorbed [23, 24]. Some studies indicated that urea generation in hepatocytes can be obstructed by insufficient adenosine triphosphate (ATP) supply and depressed levels of arginine and citrulline after exposure to hypoxia, but the genesis of creatinine is relatively constant [25]. This may be an explanation to the change of relationship between BUN and creatinine after rapid ascending to high altitude.

\section{The physiological risk factors for $\mathrm{HAH}$ at $3700 \mathrm{~m}$}

The elevation of altitude results in a lower partial pressure of oxygen in the inspired air, and $\mathrm{SpO}_{2}$ is a direct parameter that reflect the oxygen delivery. Insufficient oxygen consumption of cerebral tissue leads to function disorder and cytotoxic oedema, which is the main cause of HAH [26]. In addition, increased HR reflect the activity of sympathetic nervous system, which can lead to higher cardiac output and vasoconstriction of viscera, promote the redistribution of blood (mostly into vital organs such as brain). The accumulation of fluid in the brain result in increased intracranial pressure (ICP), which is another crucial cause of HAH [6]. Multiple lines of studies have reported that reduced $\mathrm{SpO}_{2}$ and increased $\mathrm{HR}$ are independent risk factors for $\mathrm{HAH}$, which is supported by our findings $[1,7,11]$.

\section{Blood urea nitrogen is an independent risk factor for $\mathrm{HAH}$ at both sea level and $3700 \mathrm{~m}$}

Our results first found that BUN is an independent risk factor of $\mathrm{HAH}$ at both sea level and $3700 \mathrm{~m}$, and the values of BUN at $3700 \mathrm{~m}$ positively associated with $\mathrm{HAH}$ severity. Although more evidence of this finding is lacking, there still can be some potential explanations.

The first possible explanation is that the urea concentration may reflect the oxygen supply and utilization in cells. As discussed above, the correlation between BUN and creatinine showed a good linearity at sea level but no correlation at $3700 \mathrm{~m}$, suggest that the production of

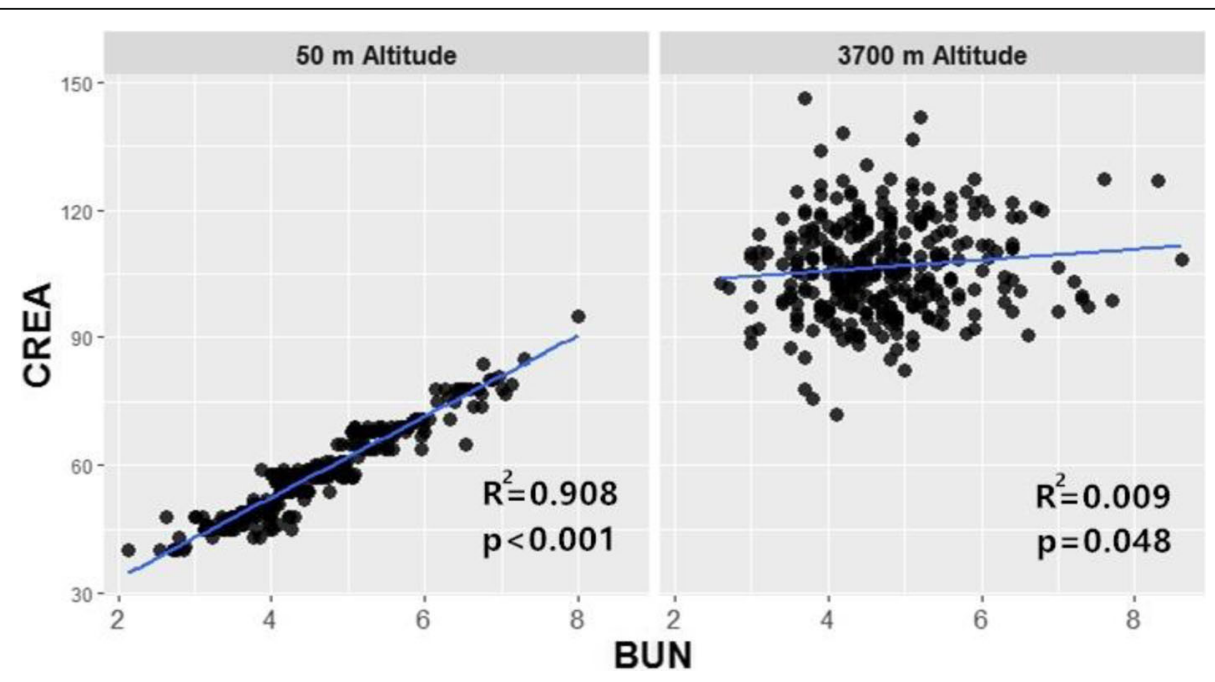

Fig. 2 the correlation between BUN and creatinine at $50 \mathrm{~m}$ and $3700 \mathrm{~m}$. BUN, Blood urea nitrogen (mmol/L); CREA, creatinine (umol/L) 
BUN may be affected by high altitude environment, and the concentration of BUN may partially reflect metabolism status and the oxygen utilization status of hepatocyte. Relative to BUN, other liver function parameters such as ALT, AST and bilirubin were aimed at substantial damage of liver and biliary tract. Some vitro experiments demonstrated that under hypobaric hypoxia, ATP was decreased in multiple cell lines, and as the oxygen concentration was decreased, production of both urea in isolated rat hepatocytes declined [25, 27, 28]. Study on the effect of acute hypoxic hypoxia on the profile of plasma amino acids in rats showed that after exposure to hypoxia for $5 \mathrm{~h}$, the concentrations of arginine and citrulline (which are related to the urea cycle) were depressed [25]. These findings implicate that the BUN concentration in plasma may reflect the oxygen-driven catabolism, and higher BUN may indicate better oxygen supply and utilization of hepatocytes, even brain cells, which is the main cause of $\mathrm{HAH}$.

The second potential explanation is that the hyperosmolar properties of urea may help with reducing intracranial pressure and brain volume [29]. Through brain imaging of patients with acute mountain sickness, some studies showed that intracellular and extracellular water accumulation influenced by increased permeability of the blood-brain barrier, resulted in cerebral swelling, sulci disappearing and changing of grey matter [6]. These verified that the inflation of brain volume and elevation of ICP is a vital sign of HAH. In 1960s, urea became the first widely used hyperosmolar compound in clinic for reducing ICP and alleviating cerebral swelling $[30,31]$. The penetrability of urea from extracellular into brain tissue is $1 / 10$ compared with the penetration into muscle, and its blood to brain transfer coefficient (a measurement of clearance) is $5 \times 10^{-3} \mathrm{ml} / \mathrm{g} / \mathrm{min}$, a value that is 3 orders of magnitude lower than water [32]. Therefore, exogenous urea can maintain certain osmotic pressure inside and outside the brain cell and prevent excessive accumulation of liquids and cerebral swelling. Because urea undergoes renal excretion, the dehydrating effect of exogenous urea on parenchyma is short lived, and the effect of endogenous urea during brain oedema have not been studied.

Another possible explanation is the products from urea cycle, nitric oxide (NO). Arginine generated as intermediate products by argininosuccinate lyase during urea cycle, and is the basic substrate of nitric oxide synthase (NOS) for generating nitric oxide. Nitric oxide has a short half-life and rapidly diffuses into the vascular smooth muscle where it affects modulation of calcium ions, mediated by cyclic guanosine monophosphate (cGMP), leading to vasodilatation [33]. They have significant effects in relieving pulmonary hypertension, improve cardiac output and blood gas exchange [34]. However, relevant studies about the correlation between ICP, pulmonary hypertension and endogenic urea are still lacking.

\section{Limitations}

Limited by field study, the time of onset of headache of participants were recorded by memories, not precisely, which should be improved in future. After the onset of headache, participants have not descent to low altitude immediately, which not strictly satisfied the criteria of the International Classification of Headache Disorders. The sample size was small, and there are many other potential risk factors that can be considered in the study, such as nitric oxide and $\mathrm{PaCO}_{2}$. The participants in our study were all young male individuals, which may limit extrapolation of our results.

\section{Conclusions}

Our study found the frequency of HAH was high (74.84\%) after acute exposure to $3700 \mathrm{~m}$. We confirmed that $\mathrm{SpO}_{2}$ and $\mathrm{HR}$ at $3700 \mathrm{~m}$ are independent risk factors for $\mathrm{HAH}$, and firstly identified the independent association between BUN and HAH at both sea level and $3700 \mathrm{~m}$, which suggested that lower BUN may be a new independent risk factor for $\mathrm{HAH}$.

\section{Additional files}

Additional file 1: The distribution and QQ-norm plot of SpO2 at $50 \mathrm{~m}$ and $3700 \mathrm{~m}$. (DOCX $168 \mathrm{~kb}$ )

Additional file 2: The incidence of mild, moderate and severe headaches after ascent to $3700 \mathrm{~m}$ altitude. (DOCX $16 \mathrm{~kb}$ )

Additional file 3: The Shapiro-Wilk normality test of parameters at $50 \mathrm{~m}$ and $3700 \mathrm{~m}$. (DOCX $12 \mathrm{~kb})$

\section{Abbreviations}

ALT: alanine aminotransferase; AMS: acute mountain sickness; AST: aspartate aminotransferase; ATP: adenosine triphosphate; BMI: body mass index; BUN: blood urea nitrogen; cGMP: cyclic guanosine monophosphate; CREA: creatinine; CRFs: Structured case report forms; DBIL: direct serum bilirubin; DBP: diastolic blood pressure; eGFR: estimated glomerular filtration rate; $\mathrm{HAH}$ : high-altitude headache; Hct: hematocrit; Hgb: hemoglobin; HR: heart rate; IBIL: indirect serum bilirubin; ICP: intracranial pressure; IMCD: inner medullary collecting duct; IQR: interquartile range; LYM: absolute lymphocyte count; LYM\%: lymphocyte percentage; MCH: mean corpuscular hemoglobin; MCV: mean corpuscular volume; MPV: mean platelet volume; NO: nitric oxide; NOS: nitric oxide synthase; OR: Odds ratio; PLT: platelet count; RBC: red blood cell count; SBP: systolic blood pressure; SD: standard deviations; SpO2: blood oxygen saturation; TBIL: total serum bilirubin; WBC: white blood cell count

\section{Acknowledgements}

The authors thank Wenyuan Duan, MD, Tongjian Wang, MD and Kai Dong, MM (Institute of Cardiovascular Disease, General Hospital of Jinan Military Region, China) for invaluable support during data collection. The authors also thank Yajun Yang, PhD, Dr. Xingdong Chen (Fudan University, China) and Ziyu Yan, Juan Zhang and Jiangli Xue (Fudan-Taizhou Institute of Health Sciences, China) for support with the data entry of this study. No one received compensation for his/her contribution. 


\section{Availability of data and materials}

Unfortunately, we cannot provide raw data and materials. As stated in the informed consent signed with all participants, we would keep all personal information and subject materials confidential, which would only been used for research purpose, published as research results or conclusions. In addition, according to the cooperation agreement, the data and materials owned by our collaborators, we only have the authority to analyze and disclose the results.

\section{Funding}

This work was supported by the National Natural Science Foundation of China (31330038, 31521003, 31460286), Shanghai Municipal Science and Technology Major Project (2017SHZDZX01), Ministry of Science and Technology (2015FY1117000), Science and Technology Committee of Shanghai Municipality (16JC1400500), the 111 Project (B13016).

\section{Authors' contributions}

$\sqcup$ were responsible for study supervision and contributed to the study concept and design, data collection, data analysis, drafting and revision of the manuscript. KW contributed to study supervision, and were responsible for data collection, data analysis, drafting and revision of the manuscript. XW, YM and YL contributed to study supervision and data collection. WP contributed to the revise of the manuscript. MZ, YW, YL and XL contributed to data analysis and interpretation, drafting and revision of the manuscript. JW contributed to study supervision, drafting and revision of the manuscript. LK contributed to the data collection. All authors read and approved the final manuscript.

\section{Ethics approval and consent to participate}

This study was approved by the Human Ethics Committee of Fudan University. All patients provided written informed consent.

\section{Consent for publication}

Not applicable.

\section{Competing interests}

The authors declare that they have no competing interests.

\section{Publisher's Note}

Springer Nature remains neutral with regard to jurisdictional claims in published maps and institutional affiliations.

\section{Author details}

'State Key Laboratory of Genetic Engineering, Collaborative Innovation Center for Genetics and Development, School of Life Sciences, Fudan University, Shanghai 200438, China. ${ }^{2}$ Ministry of Education Key Laboratory of Contemporary Anthropology, Department of Anthropology and Human Genetics, School of Life Sciences, Fudan University, Shanghai 200438, China. ${ }^{3}$ Human Phenome Institute, Fudan University, Shanghai 201203, China. ${ }^{4}$ Institute of Cardiovascular Disease, General Hospital of Jinan Military Region, Jinan 250022, Shandong, China. ${ }^{5}$ Key Laboratory of High Altitude Environment and Genes Related to Diseases of Tibet Autonomous Region, School of Medicine, Xizang Minzu University, Xianyang 712082, China. 'Six Industrial Research Institute, Fudan University, Shanghai 200433, China.

\section{Received: 18 February 2018 Accepted: 2 July 2018}

\section{Published online: 25 July 2018}

\section{References}

1. Burtscher M, Mairer K, Wille M, Broessner G (2011) Risk factors for highaltitude headache in mountaineers. Cephalalgia 31(6):706-711

2. Carod-Artal FJ (2014) High-altitude headache and acute mountain sickness. Neurologia 29(9):533-540

3. Roach RC, Bartsch P, Hackett P, Oelz O: The Lake Louise acute mountain sickness scoring system: the Lake Louise AMS scoring consensus committee; 1993

4. Headache Classification Subcommittee of the International Headache S (2004) The international classification of headache disorders: 2 nd edition. Cephalalgia 24(Suppl 1):9-160

5. Bes A, Kunkel R, Lance JW, Nappi G, Pfaffenrath V, Rose FC, Schoenberg BS, Soyka D, Tfelt-Hansen P, Welch KMA et al (2013) The international classification of headache disorders, 3rd edition (beta version). Cephalalgia 33(9):629-808

6. Wilson MH, Newman S, Imray CH (2009) The cerebral effects of ascent to high altitudes. Lancet Neurol 8(2):175-191

7. Bian SZ, Zhang JH, Gao XB, Li M, Yu J, Liu X, Dong JQ, Chen GZ, Huang L (2013) Risk factors for high-altitude headache upon acute high-altitude exposure at $3700 \mathrm{~m}$ in young Chinese men: a cohort study. J Headache Pain 14:35

8. Bian SZ, Jin J, Li QN, Yu J, Tang CF, Rao RS, Yu SY, Zhao XH, Qin J, Huang L (2015) Hemodynamic characteristics of high-altitude headache following acute high altitude exposure at $3700 \mathrm{~m}$ in young Chinese men. J Headache Pain 16:527

9. Harris NS, Wenzel RP, Thomas SH (2003) High altitude headache: efficacy of acetaminophen vs. ibuprofen in a randomized, controlled trial. J Emerg Med 24(4):383-387

10. Mampreso E, Maggioni F, Viaro F, Disco C, Zanchin G (2009) Efficacy of oxygen inhalation in sumatriptan refractory "high altitude" cluster headache attacks. J Headache Pain 10(6):465-467

11. Lawley JS (2011) Identifying the possible risk factors for high-altitude headache in mountaineers. Cephalalgia 31(16):1677-1678

12. Sutherland Al, Morris DS, Owen CG, Bron AJ, Roach RC (2008) Optic nerve sheath diameter, intracranial pressure and acute mountain sickness on Mount Everest: a longitudinal cohort study. Br J Sports Med 42(3):183-188

13. Guo WY, Bian SZ, Zhang JH, Li QN, Yu J, Chen JF, Tang CF, Rao RS, Yu SY, Jin J et al (2017) Physiological and psychological factors associated with onset of high-altitude headache in Chinese men upon acute high-altitude exposure at $3700 \mathrm{~m}$. Cephalalgia 37(4):336-347

14. Huang $H$, Liu B, Wu G, Xu G, Sun BD, Gao YQ (2017) Hematological risk factors for high-altitude headache in Chinese men following acute exposure at 3,700 m. Front Physiol 8:801

15. Jones RM, Terhaard C, Zullo J, Tenney SM (1981) Mechanism of reduced water intake in rats at high altitude. Am J Phys 240(3):R187-R191

16. Westerterp KR, Meijer EP, Rubbens M, Robach P, Richalet JP (2000) Operation Everest III: energy and water balance. Pflugers Archiv : European J Physiol 439(4):483-488

17. Bestle MH, Olsen NV, Poulsen TD, Roach R, Fogh-Andersen N, Bie P (2002) Prolonged hypobaric hypoxemia attenuates vasopressin secretion and renal response to osmostimulation in men. J Appl Physiol 92(5):1911-1922

18. Pichler J, Risch L, Hefti U, Merz TM, Turk AJ, Bloch KE, Maggiorini M, Hess T, Barthelmes D, Schoch OD et al (2008) Glomerular filtration rate estimates decrease during high altitude expedition but increase with Lake Louise acute mountain sickness scores. Acta Physiol 192(3):443-450

19. Hackett P, Rennie D (2002) High-altitude pulmonary edema. Jama 287(17): 2275-2278

20. Naeije R (2010) Physiological adaptation of the cardiovascular system to high altitude. Prog Cardiovasc Dis 52(6):456-466

21. Goldfarb-Rumyantzev AS, Alper SL (2014) Short-term responses of the kidney to high altitude in mountain climbers. Nephrol Dial Transplant 29(3):497-506

22. Shah MB, Braude D, Crandall CS, Kwack H, Rabinowitz L, Cumbo TA, Basnyat B, Bhasyal G (2006) Changes in metabolic and hematologic laboratory values with ascent to altitude and the development of acute mountain sickness in Nepalese pilgrims. Wilderness Environ Med 17(3):171-177

23. Muller F, Dommergues M, Bussieres L, LortatJacob S, Loirat C, Oury JF, Aigrain Y, Niaudet P, Aegerter P, Dumez Y (1996) Development of human renal function: reference intervals for 10 biochemical markers in fetal urine. Clin Chem 42(11):1855-1860

24. Deguchi E, Akuzawa M (1997) Renal clearance of endogenous creatinine, urea, sodium, and potassium in normal cats and cats with chronic renal failure. J Vet Med Sci 59(7):509-512

25. Kashiwagura T, Wilson DF, Erecinska M (1984) Oxygen dependence of cellular metabolism: the effect of $\mathrm{O} 2$ tension on gluconeogenesis and urea synthesis in isolated rat hepatocytes. J Cell Physiol 120(1):13-18

26. Kallenberg K, Bailey DM, Christ S, Mohr A, Roukens R, Menold E, Steiner T, Bartsch P, Knauth M (2007) Magnetic resonance imaging evidence of cytotoxic cerebral edema in acute mountain sickness. J Cereb Blood Flow Metab 27(5):1064-1071

27. Lipton P, Whittingham TS (1982) Reduced ATP concentration as a basis for synaptic transmission failure during hypoxia in the in vitro Guinea-pig hippocampus. J Physiol 325:51-65

28. Heerlein K, Schulze A, Hotz L, Bartsch P, Mairbaurl H (2005) Hypoxia decreases cellular ATP demand and inhibits mitochondrial respiration of a549 cells. Am J Respir Cell Mol Biol 32(1):44-51 
29. Otvos B, Kshettry VR, Benzel EC (2014) The history of urea as a hyperosmolar agent to decrease brain swelling. Neurosurg Focus 36(4)

30. Stubbs J, Pennybacker J (1960) Reduction of intracranial pressure with hypertonic urea. Lancet 1(7134):1094-1097

31. Matson DD (1965) Treatment of cerebral swelling. N Engl J Med 272:626-628

32. Go KG, van Woudenberg F, Woldring MG, Ebels EJ, Beks JW, Smeets EH

(1969) The penetration of 14C-urea and 3H-water into the rat brain with cold-induced cerebral oedema. Histological and autoradiographic study of the oedema. The effect of urovert. Acta Neurochir 21(2):97-122

33. Martinez-Romero R, Canuelo A, Siles E, Oliver FJ, Martinez-Lara E (2012) Nitric oxide modulates hypoxia-inducible factor-1 and poly(ADP-ribose) polymerase-1 cross talk in response to hypobaric hypoxia. J Appl Physiol 112(5):816-823

34. Pearson DL, Dawling S, Walsh WF, Haines JL, Christman BW, Bazyk A, Scott N, Summar ML (2001) Neonatal pulmonary hypertension-urea-cycle intermediates, nitric oxide production, and carbamoyl-phosphate synthetase function. N Engl J Med 344(24):1832-1838

Ready to submit your research? Choose BMC and benefit from:

- fast, convenient online submission

- thorough peer review by experienced researchers in your field

- rapid publication on acceptance

- support for research data, including large and complex data types

- gold Open Access which fosters wider collaboration and increased citations

- maximum visibility for your research: over $100 \mathrm{M}$ website views per year

At BMC, research is always in progress.

Learn more biomedcentral.com/submissions 